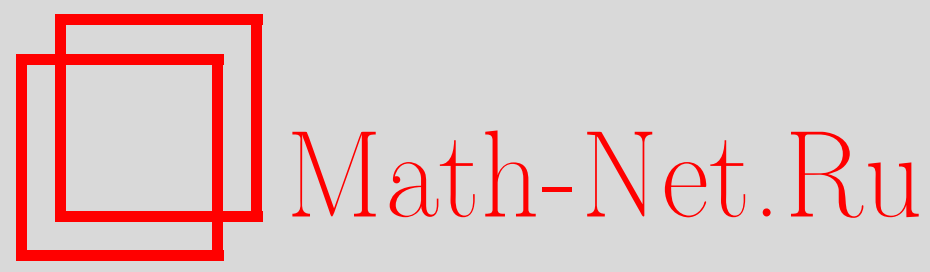

М. И. Анохин, О некоторых множествах групповых функций, Матем. заметки, 2003, том 74, выпуск 1, 3-11

DOI: https://doi.org/10.4213/mzm240

Использование Общероссийского математического портала Math-Net.Ru подразумевает, что вы прочитали и согласны с пользовательским соглашением http://www.mathnet.ru/rus/agreement

Параметры загрузки:

IP: 54.210 .77 .194

26 апреля 2023 г., 14:05:55

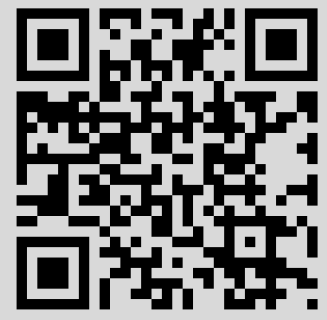




\title{
О НЕКОТОРЫХ МНОЖЕСТВАХ ГРУППОВЫХ ФУНКЦИЙ
}

\author{
М.И. Анохин
}

Пусть $G$-группа, $A$ - абелева группа и $n$-целое число такое, что $n \geqslant-1$. В работе изучаются множества $\Phi_{n}(G, A)$ всех функций из $G$ в $A$ степени не более $n$. По существу, эти множества были введены О. А. Логачёвым, А. А. Сальниковым и В. В. Ященко. Описаны все случаи, когда любая функция из $G$ в $A$ имеет ограниченную и не обязательно ограниченную конечную степень. Кроме того, показано, что если $G$ конечна, то изучение множества $\Phi_{n}(G, A)$ сводится к изучению множеств $\Phi_{n}\left(G / O^{p}(G), A_{p}\right)$ для простых чисел $p$, делящих $\left|G / G^{\prime}\right|$. Здесь $O^{p}(G)$ - $p$-корадикал группы $G, A_{p}-$ $p$-компонента группы $A$, а $G^{\prime}$ - коммутант группы $G$.

Библиография: 7 названий.

1. Введение. Пусть $G$ - произвольная группа и $A$ - произвольная абелева группа. В работе [1] Логачёв, Сальников и Ященко построили для каждого целого $n \geqslant-1$ множество функций из $G$ в $A$, которое мы обозначаем через $\Phi_{n}(G, A)$ (его определение см. в разделе 2). Отметим, что в вьшеуказанной работе это множество обозначалось через $\operatorname{RM}_{n}(G, A)$, что указьвало на его связь с $n$-м кодом Рида-Маллера. Однако по мнению автора обобщением $n$-го кода Рида-Маллера более естественно считать образ $\Phi_{n}(G, A)$ в групповом кольце $\mathbb{Z}_{e} G$ при некотором изоморфизме, который определен в случае, когда $G$ конечна и $A$ является циклической порядка $e$ (см. замечание 6$)$. Кроме того, в работе [1] групшы $G$ и $A$ предполагались конечными абелевыми.

Отметим, что $\Phi_{n}(G, A) \subseteq \Phi_{n+1}(G, A)$ для любого $n$. Как и в работе [1], каждой

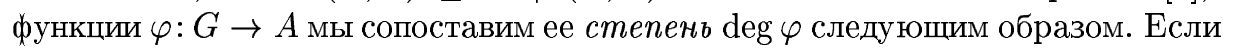

$$
\varphi \in \Phi_{*}(G, A)=\bigcup_{n=-1}^{\infty} \Phi_{n}(G, A)
$$

то $\operatorname{deg} \varphi=\min \left\{n \geqslant-1 \mid \varphi \in \Phi_{n}(G, A)\right\}$, а в противном случае $\operatorname{deg} \varphi=\infty$. Тогда $\Phi_{n}(G, A)$ при любом $n \geqslant-1$ - это множество всех функций из $G$ в $A$ степени не более $n$, а $\Phi_{*}(G, A)$ - множество всех функций из $G$ в $A$, имеющих конечные степени.

Говоря неформально, основные результаты настоящей работы заключаются в следующем. Описаны все случаи, когда любая функция из $G$ в $A$ имеет ограниченную и не обязательно ограниченную конечную степень (см. соответственно теорему 1 и теорему 2). Предположим теперь, что групша $G$ конечна. Тогда теорема 3 показьвает,

Работа выполнена при финансовой поддержке Российского фонда фундаментальных исследований, гранты № 99-01-00929 и № 99-01-00941. 
что для $n \geqslant-1$ и $n=$ * изучение множества $\Phi_{n}(G, A)$ сводится к изучению множеств $\Phi_{n}\left(G / O^{p}(G), A_{p}\right)$ при простых числах $p$, делящих $\left|G / G^{\prime}\right|$. Здесь $O^{p}(G)-p$-корадикал групшы $G$ (см. раздел 2$), A_{p}-p$-компонента группы $A$ (см. раздел 4$)$, а $G^{\prime}-$ коммутант группы $G$. Отметим, что любая функция из $G / O^{p}(G)$ в $A_{p}$ принадлежит $\Phi_{*}\left(G / O^{p}(G), A_{p}\right)$, так как $G / O^{p}(G)$ - конечная $p$-группа, а $A_{p}$ - $p$-группа (см. теорему 2). В следствии 1 приводится некоторая формула для степени произвольной функции из $\Phi_{*}(G, A)$. Получен также некоторый критерий принадлежности произвольной функции из $G$ в $A$ множеству $\Phi_{*}(G, A)$ (следствие 2$)$. Если $G$ нильпотентна, то теорема 3 может быть записана в несколько более удобном виде (см. следствие 3 ).

Автор благодарит В. В. Ященко, которьй направил его усилия на изучение объектов настоящей работы. Автор признателен О. А. Логачёву, А. А. Сальникову и В. В. Ященко, а также руководителям и участникам семинара "Кольца и модули" кафедры высшей алгебры механико-математического факультета МГУ за полезные обсуждения данной работы.

2. Определения, обозначения и необходимые факты. Как обычно, через $\mathbb{Z}$ мы будем обозначать множество (кольцо) всех целых чисел. Пусть также $\mathbb{N}_{k}=\{n \in$ $\mathbb{Z} \mid \geqslant k\}$ для произвольного $k \in \mathbb{Z}$. Если $m \in \mathbb{N}_{1}$, то через $\mathbb{Z}_{m}$ обозначается кольцо вычетов $\mathbb{Z} / m \mathbb{Z} ;$ кроме того, положим $\mathbb{Z}_{\infty}=\mathbb{Z}$. Аддитивная група кольца $\mathbb{Z}_{m}$ при $m \in$ $\mathbb{N}_{1} \cup\{\infty\}$ будет обозначаться так же, как и это кольцо. Для произвольных множеств $X$ и $Y$ через $Y^{X}$ обозначается множество всех функций из $X$ в $Y$. В работе используется постфиксная запись, т.е. значение функции $\varphi \in Y^{X}$ на элементе $x \in X$ записьвается в виде $x \varphi$, а не $\varphi(x)$. Если $\varphi \in Y^{X}$ и $\psi \in Z^{Y}$, то $\varphi \psi$ будет обозначать композицию функций $\varphi$ и $\psi$ (в этом порядке), т.е. $x(\varphi \psi)=(x \varphi) \psi$ для любого $x \in X$. Через $\langle S\rangle$ обозначается подгруппа, порожденная подмножеством $S$ некоторой группы. Если $s-$ элемент какой-либо группы, то вместо $\langle\{s\}\rangle$ мы будем писать $\langle s\rangle$.

На протяжении всей работы через $G$ будет обозначаться некоторая группа, а через $A$ - некоторая абелева групша. Для групшы $G$ мы используем мультипликативную запись, а для группы $A$ аддитивную. Если $y$ - элемент некоторого множества $Y$, то через $\bar{y}$ будет обозначаться функция из $Y^{G}$, тождественно равная $y$ на $G$.

Пусть $R$ - ассоциативное кольцо с единищей. Тогда $R G$ обозначает групповое кольцо группы $G$ над кольцом $R$. Если $H$ - нормальная подгруппа группы $G$, то через $\Delta_{H}(G, R)$ будет обозначаться ядро гомоморфизма $R G$ на $R(G / H)$, продолжающего по линейности естественньй гомоморфизм $G$ на $G / H$. Хорошо известно, что если нормальная подгруппа $H$ группы $G$ порождается множеством $S$, то $\Delta_{H}(G, R)$ порождается как левьй и как правьй идеал $R G$ множеством $\{s-1 \mid s \in S\}$. Напомним, что идеал $\Delta_{G}(G, R)$ назьвается фундаментальным идеалом кольца $R G$; мы будем обозначать этот идеал через $\Delta(G, R)$. Для $n \in \mathbb{N}_{1}$ через $\Delta^{n}(G, R)$ обозначается $n$-я степень идеала $\Delta(G, R)$; под $\Delta^{0}(G, R)$ мы понимаем $R G$. Нам потребуется следующая лемма, вытекающая из леммы 2 работы [2] или из теоремы 9 работы [3].

Лемма 1. Идеал $\Delta(G, R)$ нильпотентен тогда и только тогда, когда выполнено хотя бы одно из следующих условий:

1) $G=\{1\}$;

2) $R=\{0\}$;

3) существует простое число $p$ такое, что $G$ - конечная p-группа и элемент $р$ кольиа $R$ нильпотентен.

Множество $A^{G}$ является абелевой группой относительно операции поточечного сло- 
жения. Кроме того, групша $G$ действует справа на $A^{G}$ следующим образом:

$$
x(\varphi g)=\left(x g^{-1}\right) \varphi, \quad \varphi \in A^{G}, \quad x, g \in G .
$$

Через $е$ всюду в настоящей работе будет обозначаться экспонента групшы $A$ (возможно, бесконечная), которая совпадает с экспонентой групшы $A^{G}$. Очевидно, что група $A^{G}$ является точным унитарным правым $\mathbb{Z}_{e} G$-модулем относительно действия $\mathbb{Z}{ }_{e} G$, продолжающего по линейности действие (1).

Определим множества $\Phi_{n}(G, A) \subseteq A^{G}$ индукцией по $n \in \mathbb{N}_{-1}$ следующим образом:

$$
\Phi_{-1}(G, A)=\{\overline{0}\}, \quad \Phi_{n}(G, A)=\left\{\varphi \in A^{G} \mid \varphi \Delta\left(G, \mathbb{Z}_{e}\right) \subseteq \Phi_{n-1}(G, A)\right\}, \quad n \in \mathbb{N}_{0} .
$$

Очевидно, что $\Phi_{n}(G, A)$ при любом $n \in \mathbb{N}_{-1}$ является подмодулем $A^{G}$ и

$$
\Phi_{-1}(G, A) \subseteq \Phi_{0}(G, A) \subseteq \ldots .
$$

По существу, множества $\Phi_{n}(G, A)$ были определены в работе [1], хотя там группы $G$ и $A$ предполагались конечными абелевыми и вместо $\Phi_{n}(G, A)$ использовалось обозначение $\mathrm{RM}_{n}(G, A)$.

ЗАмЕчаниЕ 1 . Пусть $\{\overline{0}\}=M_{-1} \subseteq M_{0} \subseteq \ldots-$ ряд подмодулей $\mathbb{Z}_{e} G$-модуля $A^{G}$, в факторах которого групп $G$ действует тождественно (другими словами, $M_{n} \Delta\left(G, \mathbb{Z}_{e}\right) \subseteq$ $M_{n-1}$ для всех $\left.n \in \mathbb{N}_{0}\right)$. Тогда индукция по $n$ дает включение $M_{n} \subseteq \Phi_{n}(G, A)$ для любого $n \in \mathbb{N}_{-1}$. Это свойство ряда (2) показьвает, что он является аналогом верхнего центрального ряда в группе.

Лемма 2. Пусть $\varphi \in A^{G} u n \in \mathbb{N}_{-1}$. Тогда следующие условия әквивалентны:

1) $\varphi \in \Phi_{n}(G, A)$;

2) $\varphi \Delta^{n+1}\left(G, \mathbb{Z}_{e}\right)=\{\overline{0}\}$

3) $\sum_{i_{1}, \ldots, i_{k}}(-1)^{n+1-k}\left(x y_{i_{1}} \ldots y_{i_{k}}\right) \varphi=0$ для всех $x, y_{1}, \ldots, y_{n+1} \in G$, где суммирование производится по всевозможным последовательностям иелых чисел $i_{1}, \ldots, i_{k}$ таким, что $0 \leqslant k \leqslant n+1 u 1 \leqslant i_{1}<\cdots<i_{k} \leqslant n+1$.

Эквивалентность условий 1 и 2 леммы 2 легко доказьвается индукцией по $n$. Эквивалентность условий 2 и 3 этой леммы следует из того, что $\Delta^{n+1}\left(G, \mathbb{Z}_{e}\right)$ порождается как правый идеал $\mathbb{Z}_{e} G$ множеством всех элементов вида $\left(g_{1}-1\right) \ldots\left(g_{n+1}-1\right)$, где $g_{1}, \ldots, g_{n+1} \in G$. Отметим, что $\Delta^{n+1}\left(G, \mathbb{Z}_{e}\right)$ порождается вьшеуказанным множеством также и как левьй идеал $\mathbb{Z}_{e} G$, а при $n \in \mathbb{N}_{0}$ - даже как подгруппа аддитивной группы $\mathbb{Z}_{e} G$.

Положим

$$
\Phi_{*}(G, A)=\bigcup_{n=-1}^{\infty} \Phi_{n}(G, A) .
$$

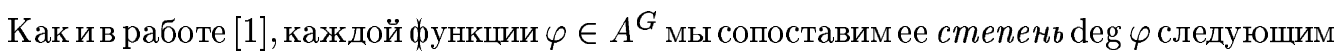
образом:

$$
\operatorname{deg} \varphi= \begin{cases}\min \left\{n \in \mathbb{N}_{-1} \mid \varphi \in \Phi_{n}(G, A)\right\}, & \text { если } \varphi \in \Phi_{*}(G, A) ; \\ \infty, & \text { если } \varphi \notin \Phi_{*}(G, A) .\end{cases}
$$

Тогда $\Phi_{n}(G, A)$ при любом $n \in \mathbb{N}_{-1}$ - это множество всех функций из $A^{G}$ степени не более $n$, а $\Phi_{*}(G, A)$ - множество всех функций из $A^{G}$, имеющих конечные степени.

Пусть $p$ - простое число. Через $O^{p}(G)$ будет обозначаться $p$-корадикал групшы $G$, т.е. наименьшая подгруппа в множестве всех нормальных подгрупп $H$ группы $G$ таких, что $G / H$ является $p$-группой. Разумеется, не всякая группа обладает $p$-корадикалом. Однако $O^{p}(G)$ заведомо существует, если $G$ - конечная группа или периодическая абелева группа. 
Лемма 3. Предположим, что әруппа $G$ конечна. Пусть $p$ - простое число $u R$ - ассоциативное кольцо с единицей, в котором әлемент $p$ нильпотентен. Пусть также $l=\min \left\{n \in \mathbb{N}_{0} \mid \Delta^{n}\left(G / O^{p}(G), R\right)=\{0\}\right\}$ (әто число определено ввиду леммы 1). Тогда

$$
\Delta_{O^{p}(G)}(G, R)=\Delta^{l}(G, R)=\Delta^{l+1}(G, R)=\ldots .
$$

ДокАЗАТЕЛьСТво. Обозначим через $S$ множество всех элементов групшы $G$, порядки которых взаимно просты с $p$. Пусть $s \in S$ и $m$ - порядок $s$. Тогда согласно формуле бинома

$$
1=((s-1)+1)^{m}=1+m(s-1)+\left(\sum_{i=0}^{m-2}\left(\begin{array}{c}
m \\
i+2
\end{array}\right)(s-1)^{i}\right)(s-1)^{2} .
$$

Следовательно, $m(s-1)$ делится на $(s-1)^{2}$ в кольце $R\langle s\rangle$. Ввиду взаимной простоты $m$ и $p$ отсюда вытекает, что $s-1=\alpha(s-1)^{2}$ для некоторого $\alpha \in R\langle s\rangle$. Из последнего равенства с помошью индукции по $n$ легко получить, что $s-1=\alpha^{n}(s-1)^{n+1}$ для любого $n \in \mathbb{N}_{0}$. В частности, $s-1 \in \Delta^{l+1}(G, R)$. Легко видеть, что $\langle S\rangle=O^{p}(G)$, поэтому $\Delta_{O^{p}(G)}(G, R)$ порождается как левьй и как правый идеал $R G$ множеством $\{s-$ $1 \mid s \in S\} \subseteq \Delta^{l+1}(G, R)$. Следовательно,

$$
\Delta_{O^{p}(G)}(G, R) \subseteq \Delta^{l+1}(G, R) .
$$

С другой стороны,

$$
\Delta^{l}(G, R) \subseteq \Delta_{O^{p}(G)}(G, R)
$$

так как $\Delta^{l}\left(G / O^{p}(G), R\right)=\{0\}$ и $\Delta_{O^{p}(G)}(G, R)$ - ядро гомоморфизма $R G$ на $R\left(G / O^{p}(G)\right)$, продолжающего по линейности естественньй гомоморфизм $G$ на $G / O^{p}(G)$. Утверждение леммы вытекает из включений (3) и (4) и тривиального включения $\Delta^{l+1}(G, R) \subseteq \Delta^{l}(G, R)$. Лемма доказана.

\section{3. Общие свойства множеств $\Phi_{n}(G, A)$.}

ЗАмечАниЕ 2 . Пусть $A$ является подгрупой некоторой абелевой грушыы $B$. Тогда из эквивалентности условий 1 и 3 леммы 2 вытекает, что $\Phi_{n}(G, A)=\Phi_{n}(G, B) \cap A^{G}$ для любого $n \in \mathbb{N}_{-1}$ (а следовательно, и для $n=*$ ). Поэтому если $\varphi \in A^{G}$, то степени $\varphi$ как функции из $A^{G}$ и как функции из $B^{G}$ совпадают.

ЗАмечание 3 . Пусть $\eta$ - гомоморфизм $A$ в некоторую абелеву группу $B$. Тогда из эквивалентности условий 1 и 3 леммы 2 вытекает, что если $\varphi \in \Phi_{n}(G, A)$, то $\varphi \eta \in$ $\Phi_{n}(G, B)$ для любого $n \in \mathbb{N}_{-1}$ (а следовательно, и для $n=*$ ). Другими словами, $\operatorname{deg} \varphi \eta \leqslant \operatorname{deg} \varphi$ для любой функции $\varphi \in A^{G}$.

ЗАмЕчаниЕ 4 . Пусть $\eta$ - гомоморфизм $G$ в некоторую группу $H$ и $\varphi \in A^{H}$. Тогда из эквивалентности условий 1 и 3 леммы 2 вытекает, что

$$
\varphi \in \Phi_{n}(H, A) \Longrightarrow \eta \varphi \in \Phi_{n}(G, A)
$$

для любого $n \in \mathbb{N}_{-1}$ (а следовательно, и для $n=*$ ). Другими словами, $\operatorname{deg} \eta \varphi \leqslant \operatorname{deg} \varphi$ для любой функции $\varphi \in A^{G}$. Если $G \eta=H$, то из вьшеуказанной эквивалентности вытекает, что

$$
\varphi \in \Phi_{n}(H, A) \Longleftrightarrow \eta \varphi \in \Phi_{n}(G, A)
$$

для любого $n \in \mathbb{N}_{-1}$ (а следовательно, и для $n=*$ ). Другими словами, $\operatorname{deg} \eta \varphi=\operatorname{deg} \varphi$ для любой функции $\varphi \in A^{G}$. 
ЗАмечание 5. Легко видеть (см. также [1]), что $\Phi_{0}(G, A)$ есть множество всех функций-констант из $A^{G}$, а $\Phi_{1}(G, A)$ совпадает с множеством всех функций вида $\eta+\bar{a}$, где $\eta$ - гомоморфизм из $G$ в $A$ и $a \in A$.

ЗАмечание 6 . Предположим, что групша $G$ конечна, а группа $A$ является циклической порядка $e \in \mathbb{N}_{1} \cup\{\infty\}$. Для удобства мы считаем, что $A$ - аддитивная группа кольца $\mathbb{Z}_{e}$, которая будет обозначаться так же, как и это кольцо. Очевидно, что функция

$$
\varphi \mapsto \sum_{g \in G}(g \varphi) g, \quad \varphi \in \mathbb{Z}_{e}^{G}
$$

является изоморфизмом $\mathbb{Z}_{e}^{G}$ и $\mathbb{Z}_{e} G$ как правых $\mathbb{Z}_{e} G$-модулей. Поэтому вместо функций из $\mathbb{Z}_{e}^{G}$ можно работать с их образами при изоморфизме (5). Для $n \in \mathbb{N}_{-1} \cup\{*\}$ образ модуля $\Phi_{n}\left(G, \mathbb{Z}_{e}\right)$ при изоморфизме (5) мы будем обозначать через $\operatorname{RM}_{n}\left(G, \mathbb{Z}_{e}\right)$. Выбор такого обозначения объясняется тем, что $\operatorname{RM}_{n}\left(G, \mathbb{Z}_{e}\right)$ при некоторых $n \in \mathbb{N}_{-1}$ является обобщением $n$-го кода Рида-Маллера. Это будет показано в замечании 7.

Пусть $n \in \mathbb{N}_{-1}$. Тогда из эквивалентности условий 1 и 2 леммы 2 вытекает, что $\operatorname{RM}_{n}\left(G, \mathbb{Z}_{e}\right)$ является левым аннулятором идеала $\Delta^{n+1}\left(G, \mathbb{Z}_{e}\right)$ в $\mathbb{Z}_{e} G$. Известно, что левый аннулятор любого двустороннего идеала в кольце $\mathbb{Z}_{e} G$ совпадает с правым аннулятором этого идеала (см. [4, теорема 3.1.2, п. (c)] для симметрических алгебр над полями). Поэтому $\operatorname{RM}_{n}\left(G, \mathbb{Z}_{e}\right)$ равен также правому аннулятору идеала $\Delta^{n+1}\left(G, \mathbb{Z}_{e}\right)$ в $\mathbb{Z}_{e} G$.

ЗАмЕчАнИЕ 7. Предположим, что $G$ - конечная $p$-группа для некоторого простого числа $p$. Тогда идеал $\Delta\left(G, \mathbb{Z}_{p}\right)$ совпадает с радикалом Джекобсона (или, что в данном случае эквивалентно, с наибольшим нильпотентным идеалом) кольца $\mathbb{Z} p($ (см. $[5$, теорема 1.2], [4, теорема 3.1 .9$, п. (а)], а также лемму 1$).$ В частности, идеал $\Delta\left(G, \mathbb{Z}_{p}\right)$ нильпотентен. Поэтому определено число $d=\max \left\{n \in \mathbb{N}_{0} \mid \Delta^{n}\left(G, \mathbb{Z}_{p}\right) \neq\{0\}\right\}$.

Пусть $n \in \mathbb{N}_{-1}$. Согласно замечанию $6 \operatorname{RM}_{n}\left(G, \mathbb{Z}_{p}\right)$ совпадает с левым (или правым) аннулятором идеала $\Delta^{n+1}\left(G, \mathbb{Z}_{p}\right)$ в $\mathbb{Z}_{p} G$. Известно (см. [6], [4, теоремы 4.2.2, 3.2.2]), что при $n \in\{-1, \ldots, d\}$ этот аннулятор равен $\Delta^{d-n}\left(G, \mathbb{Z}_{p}\right)$. Следовательно,

$$
\operatorname{RM}_{n}\left(G, \mathbb{Z}_{p}\right)= \begin{cases}\Delta^{d-n}\left(G, \mathbb{Z}_{p}\right), & \text { если } n \leqslant d, \\ \mathbb{Z}_{p} G, & \text { если } n>d .\end{cases}
$$

Степени $\Delta\left(G, \mathbb{Z}_{p}\right)$ хорошо изучены в литературе (см. [5], [4, раздел 3.3] и для случая, когда група $G$ абелева, - [7, раздел 1]). Мы приведем здесь в качестве примера один результат, который легко получить непосредственно (см. также [7, раздел 1]).

Пусть $G$ является прямьм произведением семейства $\left\{C_{1}, \ldots, C_{m}\right\}$ своих подгрупп, где $m \in \mathbb{N}_{1}$ и $C_{i}$ - циклическая группа порядка $p^{k_{i}}$ при некотором $k_{i} \in \mathbb{N}_{1}$. Пусть также $c_{i}$ - некоторый порождаюший элемент группы $C_{i}$. Тогда множество

$$
\left\{\left(c_{1}-1\right)^{r_{1}} \ldots\left(c_{m}-1\right)^{r_{m}} \mid r_{i} \in\left\{0, \ldots, p^{k_{i}}-1\right\}, r_{1}+\cdots+r_{m} \geqslant l\right\}
$$

образует базис $\Delta^{l}\left(G, \mathbb{Z}_{p}\right)\left(l \in \mathbb{N}_{0}\right)$. В частности,

$$
d=\sum_{i=1}^{m}\left(p^{k_{i}}-1\right)
$$

Отметим, что если $G$ - элементарная абелева $p$-группа (т.е. $k_{1}=\cdots=k_{m}=1$ ), то при $n \leqslant m(p-1) n$-й обобщенный код Рида-Маллера длины $p^{m}$ надполем $\mathbb{Z}_{p}$ определяется в книге [4] (определение и теорема 3.3.12) как $\Delta^{m(p-1)-n}\left(G, \mathbb{Z}_{p}\right)$. Формулы (6) и (7) показывают, что этот код совпадает с $\operatorname{RM}_{n}\left(G, \mathbb{Z}_{p}\right)$. Аналогичная ситуация имеет место и для классических бинарных кодов Рида-Маллера (частньй случай обобщенных кодов Рида-Маллера при $p=2$ ). 
Tеорема 1. Равенство $\Phi_{n}(G, A)=A^{G}$ при некотором $n \in \mathbb{N}_{-1}$ справедливо тогда и только тогда, когда идеал $\Delta\left(G, \mathbb{Z}_{e}\right)$ нильпотентен, что в свою очередь әквивалентно дизвюнкиии следующих условий:

1) $G=\{1\}$;

2) $e=1$ (другими словами, $A=\{0\})$;

3) существует простое число р такое, что $G$ - конечная $p$-группа и е является степенью $р$ (последнее условие на число е выполняется, если и только если $A$ p-группа конечной әкспоненты).

ДОКАЗАТЕЛЬСТво. Очевидно, что

$$
\Phi_{n}(G, A)=A^{G} \Longleftrightarrow A^{G} \Delta^{n+1}\left(G, \mathbb{Z}_{e}\right)=\{\overline{0}\} \Longleftrightarrow \Delta^{n+1}\left(G, \mathbb{Z}_{e}\right)=\{0\}
$$

для любого $n \in \mathbb{N}_{-1}$ ввиду эквивалентности условий 1 и 2 леммы 2 и точности $\mathbb{Z}_{e} G$-модуля $A^{G}$. Поэтому $\Phi_{n}(G, A)=A^{G}$ при некотором $n \in \mathbb{N}_{-1}$ тогда и только тогда, когда идеал $\Delta\left(G, \mathbb{Z}_{e}\right)$ нильпотентен. Эквивалентность нильпотентности идеала $\Delta\left(G, \mathbb{Z}_{e}\right)$ и дизъюнкции условий 1-3 вытекает из леммы 1 . Теорема доказана.

Теорема 2. Равенство $\Phi_{*}(G, A)=A^{G}$ справедливо тогда и только тогда, когда выполнено хотя бы одно из следующих условий:

1) $G=\{1\}$;

2) $A=\{0\}$;

3) существует простое число $p$ такое, что $G$ - конечная $p$-группа $u A$ p-zpynna.

ДокАЗАтЕльство. Предположим сначала, что $\Phi_{*}(G, A)=A^{G}$ и условия 1 и 2 не выполнены. Покажем, что в этом случае выполнено условие 3 . Выберем произвольный элемент $a \in A \backslash\{0\}$ и обозначим через $k$ его порядок (вообще говоря, бесконечный). Определим функцию $\xi \in\langle a\rangle^{G}$ следующим образом:

$$
x \xi=\left\{\begin{array}{ll}
a, & \text { если } x=1, \\
0, & \text { если } x \neq 1,
\end{array} \quad x \in G .\right.
$$

Тогда $\xi \in \Phi_{n}(G,\langle a\rangle)$ для некоторого $n \in \mathbb{N}_{-1}$ ввиду равенства $\Phi_{*}(G, A)=A^{G}$ и замечания 2. Поэтому $\xi \Delta^{n+1}\left(G, \mathbb{Z}_{k}\right)=\{\overline{0}\}$, так как условия 1 и 2 леммы 2 эквивалентны. Легко видеть, что если $\xi \alpha=\overline{0}$, где $\alpha \in \mathbb{Z}_{k} G$, то $\alpha=0$. Следовательно, $\Delta^{n+1}\left(G, \mathbb{Z}_{k}\right)=\{0\}$ и идеал $\Delta\left(G, \mathbb{Z}_{k}\right)$ нильпотентен. Ввиду леммы $1 G$ является конечной $p$-группой и $k-$ степенью $p$ для некоторого единственного и не зависящего от $a$ (так как $G \neq\{1\}$ ) простого числа $p$. Это число $p$ будет определено, так как хотя бы один элемент $a \in A \backslash\{0\}$ существует. Условие 3 вытекает теперь из произвольности выбора элемента $a$.

Пусть теперь вьполнено хотя бы одно из условий 1-3. Если выполнено условие 1 или условие 2 , то равенство $\Phi_{*}(G, A)=A^{G}$ вытекает из теоремы 1 . Предположим теперь, что выполнено условие 3 . Выберем произвольную функцию $\varphi \in A^{G}$. Тогда $\langle G \varphi\rangle-$ конечная $p$-группа. Ввиду теоремы $1 \Phi_{n}(G,\langle G \varphi\rangle)=\langle G \varphi\rangle^{G}$ для некоторого $n \in \mathbb{N}_{-1}$ (вообще говоря, зависящего от $\varphi$ ). Следовательно, $\varphi \in \Phi_{n}(G, A) \subseteq \Phi_{*}(G, A)$ (см. замечание 2). Из этого вытекает равенство $\Phi_{*}(G, A)=A^{G}$, так как функция $\varphi$ выбиралась произвольно. Теорема доказана. 
4. Свойства множеств $\Phi_{n}(G, A)$ в случае, когда группа $G$ конечна. В настоящем разделе мы будем предполагать, что группа $G$ конечна. Пусть $P$ - множество всех простых чисел $p$ таких, что $O^{p}(G) \neq G$. Легко видеть, что $P$ совпадает с множеством всех простых делителей $\left|G / G^{\prime}\right|$, где $G^{\prime}$ - коммутант групшы $G$. Для простого числа $p$ через $\omega_{p}$ мы будем обозначать естественный гомоморфизм $G$ на $G / O^{p}(G)$, а через $A_{p}-$ $p$-компоненту групшы $A$, т.е. множество всех элементов групшы $A$, порядки которых являются степенями $p$ (очевидно, что это множество является подгруппой групшы $A$ ). Пусть также $A_{P}$ - (прямая) сумма семейства $\left\{A_{p} \mid p \in P\right\}$ подгрупп группы $A$ и $T$ - некоторое множество представителей всех смежных классов $A$ по $A_{P}$, содержашее 0 . Если $n \in \mathbb{N}_{-1} \cup\{*\}$, то положим

$$
T_{n}=\left\{t \in T \mid \bar{t} \in \Phi_{n}(G, A)\right\}= \begin{cases}\{0\}, & \text { если } n=-1, \\ T, & \text { если } n \in \mathbb{N}_{0} \cup\{*\}\end{cases}
$$

(см. замечание 5).

Лемма 4. Пусть $\varphi \in A^{G} u n \in \mathbb{N}_{-1} \cup\{*\}$. Тогда $\varphi \in \Phi_{n}(G, A)$, если и только если $\varphi=\bar{t}+\psi$ для некоторых $t \in T_{n} u \psi \in \Phi_{n}\left(G, A_{P}\right)$, причем в этом случае $t u$ $\psi$, удовлетворяюшие вышеуказанным условиям, определяются по ч однозначно.

ДоказАтельство. Пусть $\varphi \in \Phi_{n}(G, A)$. Докажем существование $t$ и $\psi$, требуемых в лемме. Если $n=-1$, то $\varphi=\overline{0}$ и можно положить $t=0$ и $\psi=\overline{0}$. Предположим теперь, что $n \in \mathbb{N}_{0} \cup\{*\}$. Обозначим через $\nu$ естественньй гомоморфизм $A$ на $A / A_{P}$. Тогда $\varphi \nu \in$ $\Phi_{n}\left(G, A / A_{P}\right)$ согласно замечанию 3 . Из замечания 5 вытекает, что $\Phi_{n}\left(G, A / A_{P}\right)$ есть множество всех функций-констант из $\left(A / A_{P}\right)^{G}$, так как не существует гомоморфизмов из $G$ в $A / A_{P}$, кроме $\overline{0}$. Поэтому $\varphi \nu=\bar{t} \nu$ для некоторого $t \in T$. Очевидно, что этот элемент $t$ и функция $\psi=\varphi-\bar{t} \in \Phi_{n}(G, A) \cap A_{P}^{G}=\Phi_{n}\left(G, A_{P}\right)$ (см. замечание 2) удовлетворяют условиям леммы.

Обратно, если $\varphi=\bar{t}+\psi$ для некоторых $t \in T_{n}$ и $\psi \in \Phi_{n}\left(G, A_{P}\right)$, то $\varphi \in \Phi_{n}(G, A)$, так как $\bar{t} \in \Phi_{n}(G, A)$ и $\psi \in \Phi_{n}(G, A)$ (см. замечание 2). Единственность $t$ и $\psi$, удовлетворяющих вышеуказанным условиям (если такие $t$ и $\psi$ существуют), проверяется непосредственно. Лемма доказана.

ЗАмЕчаниЕ 8. Предположим, что группа $A$ является прямой суммой семейства $\{A(i) \mid i \in I\}$ своих подгрупп (здесь $I$-некоторое множество). Для произвольного $i \in I$ обозначим через $\pi_{i}$ проекцию $A$ на $A(i)$ относительно вышеуказанного разложения $A$ в прямую сумму. Тогда легко видеть, что $\mathbb{Z}_{e} G$-модуль $A^{G}$ является прямой суммой семейства $\left\{A(i)^{G} \mid i \in I\right\}$ своих подмодулей, причем для любого $i \in I$ функция $\varphi \mapsto \varphi \pi_{i}$ $\left(\varphi \in A^{G}\right)$ является проекцией $A^{G}$ на $A(i)^{G}$ относительно вьшеуказанного разложения $A^{G}$ в прямую сумму. Кроме того, если $\varphi \in A^{G}$ и $n \in \mathbb{N}_{-1}$, то из эквивалентности условий 1 и 3 леммы 2 вытекает, что

$$
\varphi \in \Phi_{n}(G, A) \Longleftrightarrow \varphi \pi_{i} \in \Phi_{n}(G, A(i)) \quad \text { для всех } i \in I .
$$

Следовательно, эквивалентность (8) справедлива и при $n=*$. Таким образом, $\Phi_{n}(G, A)$ является прямой суммой семейства $\left\{\Phi_{n}(G, A(i)) \mid i \in I\right\}$ для любого $n \in \mathbb{N}_{-1} \cup\{*\}$.

Лемма 5. Предположим, что $А$ является $p$-группой для некоторого простого

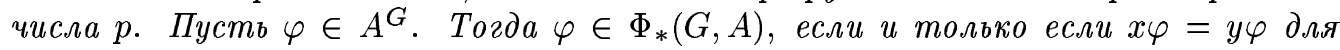
любых $x, y \in G$ таких, ито $x \equiv y\left(\bmod O^{p}(G)\right)$. 
ДокАЗАТЕльСтво. Ввиду замечания $2 \varphi \in \Phi_{*}(G, A)$, если и только если $\varphi \in \Phi_{*}(G$, $\langle G \varphi\rangle)$. Здесь $\langle G \varphi\rangle$ - конечная $p$-групша, экспоненту которой мы обозначим через $k$. В свою очередь, из леммы 3 и эквивалентности условий 1 и 2 леммы 2 следует, что $\varphi \in \Phi_{*}(G,\langle G \varphi\rangle)$ тогда и только тогда, когда $\varphi \Delta_{O^{p}(G)}\left(G, \mathbb{Z}_{k}\right)=\{\overline{0}\}$, так как $k$ является степенью $p$. Наконец, непосредственно проверяется, что последнее равенство справедливо в том и только том случае, когда для любых $x, y \in G$ из сравнения $x \equiv y$ $\left(\bmod O^{p}(G)\right)$ следует равенство $x \varphi=y \varphi$. Лемма доказана.

Из замечания 4 и леммы 5 непосредственно вытекает

Лемма 6. Предположим, что $А$ является $p$-группой для некоторого простого числар. Пусть $n \in \mathbb{N}_{-1} \cup\{*\}$. Тогда функиия $\varphi \mapsto \omega_{p} \varphi\left(\varphi \in A^{G / O^{p}(G)}\right)$ инвективно oтображсает $\Phi_{n}\left(G / O^{p}(G), A\right)$ на $\Phi_{n}(G, A)$.

Роль вьшеприведенных результатов настоящего раздела заключается в следующем. Пусть $n \in \mathbb{N}_{-1} \cup\{*\}$. Тогда лемма 4 сводит изучение множества $\Phi_{n}(G, A)$ к изучению множества $\Phi_{n}\left(G, A_{P}\right)$. Из замечания 8 следует, что изучение $\Phi_{n}\left(G, A_{P}\right)$ сводится к изучению $\Phi_{n}\left(G, A_{p}\right)$ для $p \in P$. Наконец, лемма 6 (доказательство которой использует лемму 5) позволяет свести изучение $\Phi_{n}\left(G, A_{p}\right)$ к изучению $\Phi_{n}\left(G / O^{p}(G), A_{p}\right)$ для каждого $p \in P$. Из этих трех результатов вытекает

Tеорема 3. Пусть $\varphi \in A^{G} u n \in \mathbb{N}_{-1} \cup\{*\}$. Тогда $\varphi \in \Phi_{n}(G, A)$, если и только если

$$
\varphi=\bar{t}+\sum_{p \in P} \omega_{p} \varphi_{p}
$$

для некоторых $t \in T_{n}$ u $\varphi_{p} \in \Phi_{n}\left(G / O^{p}(G), A_{p}\right)$, причем в этом случае $t$ и $\varphi_{p}$, удовлетворяющие вышеуказанным условиям, определяются по ч однозначно.

Используя описание множеств $\Phi_{n}(G, A)$, данное в теореме 3 , легко получить

СлЕДСТВИЕ 1. Пусть $\varphi \in \Phi_{*}(G, A)$. Тогда

$$
\operatorname{deg} \varphi=\max \left\{\operatorname{deg} \bar{t}, \operatorname{deg} \varphi_{p} \mid p \in P\right\}
$$

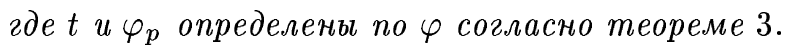

ЗАмечаниЕ 9 . Пусть $n \in \mathbb{N}_{-1} \cup\{*\}$. Тогда теорема 3 сводит изучение множества $\Phi_{n}(G, A)$ к изучению множеств $\Phi_{n}\left(G / O^{p}(G), A_{p}\right)$ для $p \in P$. Если группа $A_{p}$ при некотором $p \in P$ является прямой суммой семейства $\left\{A_{p}(i) \mid i \in I\right\}$ своих циклических подгрупп (где $I$ - некоторое множество), то ввиду замечания 8 изучение $\Phi_{n}\left(G / O^{p}(G)\right.$, $\left.A_{p}\right)$ сводится к изучению $\Phi_{n}\left(G / O^{p}(G), A_{p}(i)\right)$ для $i \in I$. В общем случае произвольная функция $\varphi \in A_{p}^{G / O^{p}(G)}(p \in P)$ принадлежит $\left\langle\left(G / O^{p}(G)\right) \varphi\right\rangle^{G / O^{p}(G)}$, где $\left\langle\left(G / O^{p}(G)\right) \varphi\right\rangle$ является конечной $p$-группой и, следовательно, разложима в прямую сумму циклических подгрупш. В свою очередь, изучение $\Phi_{n}\left(G / O^{p}(G), A_{p}(i)\right)$, где $A_{p}(i)$ - циклическая $p$-группа, сводится к изучению $\operatorname{RM}_{n}\left(G / O^{p}(G), \mathbb{Z}_{\left|A_{p}(i)\right|}\right)$ (см. замечание 6).

Согласно теореме $3 \varphi \in \Phi_{*}(G, A)$, если и только если $\varphi=\bar{t}+\sum_{p \in P} \omega_{p} \varphi_{p}$ для некоторых $t \in T$ и $\varphi_{p} \in \Phi_{*}\left(G / O^{p}(G), A_{p}\right)=A_{p}^{G / O^{p}(G)}$ (последнее равенство вытекает из теоремы 2). Кроме того, $O^{p}\left(A_{P}\right)$ равен (прямой) сумме семейства $\left\{A_{q} \mid q \in P \backslash\{p\}\right\}$ для любого простого числа $p$. Из этого нетрудно вывести 
СлеДСТВИЕ 2. Пусть $\varphi \in A^{G}$. Тогда $\varphi \in \Phi_{*}(G, A)$, если и только если выполнены два следуюших условия:

1) $x \varphi \equiv y \varphi\left(\bmod A_{P}\right)$ для любих $x, y \in G$;

2) $x \varphi \equiv y \varphi\left(\bmod O^{p}\left(A_{P}\right)\right)$ для любыx $p \in P u x, y \in G \operatorname{ma\kappa ux,~что~} x \equiv y$ $\left(\bmod O^{p}(G)\right)$.

Предположим теперь, что групша $G$ нильпотентна. Для произвольного простого числа $p$ обозначим через $G_{p}$ (единственную) силовскую $p$-подгруппу группы $G$. Тогда $G$ является прямым произведением семейства $\left\{G_{p} \mid p \in P\right\}$, так как если $G_{p} \neq\{1\}$ для некоторого простого числа $p$, то $O^{p}(G) \neq G$ и, следовательно, $p \in P$. Очевидно, что $O^{p}(G)$ равен прямому произведению семейства $\left\{G_{q} \mid q \in P \backslash\{p\}\right\}$ для любого простого числа $p$. Поэтому для произвольного $p \in P$ существует изоморфизм $\iota_{p}: G / O^{p}(G) \rightarrow G_{p}$ такой, что $\omega_{p} \iota_{p}-$ проекция $G$ на $G_{p}$. Из сказанного выше ввиду теоремы 3 и замечания 4 вытекает

СлЕДСТВИЕ 3. Предположим, что әруппа $G$ нильпотентна. Пусть $\varphi \in A^{G} u$ $n \in \mathbb{N}_{-1} \cup\{*\}$. Тогда $\varphi \in \Phi_{n}(G, A)$, если и только если существуют әлемент $t \in T_{n}$ и семейство функций $\left\{\varphi_{p} \mid p \in P\right\}$ такие, что $\varphi_{p} \in \Phi_{n}\left(G_{p}, A_{p}\right)$ для всех $p \in P u$

$$
\left(\prod_{p \in P} x_{p}\right) \varphi=t+\sum_{p \in P} x_{p} \varphi_{p}
$$

при любьх $x_{p} \in G_{p}$ (здесь через $G_{p}$ обозначена единственная силовская $p$-подгруппа әруппь $G)$.

\section{СПИСОК ЦИТИРОВАННОЙ ЛИТЕРАТУРЫ}

[1] Логачёв О.А., Сальников А.А., Ященко В. В. Некоторые характеристики "нелинейности" групповых отображений // Дискретный анализ и исследование операций. (В печати).

[2] Лихтман А. И. О групповых кольцах $p$-групп // Изв. АН СССР. Сер. матем. 1963. Т. 27. № 4. C. $795-800$.

[3] Connell I. G. On the group ring // Canad. J. Math. 1963. V. 15. № 4. P. 650-685.

[4] Zimmermann K.-H. Beiträge zur algebraischen Codierungstheorie mittels modularer Darstellungstheorie. Bayreuther mathematische Schriften, Heft 48, 1994.

[5] Jennings S. A. The structure of the group ring of a $p$-group over a modular field // Trans. Amer. Math. Soc. 1941. V. 50. №1. P. 175-185.

[6] Hill E. T. The annihilator of radical powers in the modular group ring of a $p$-group // Proc. Amer. Math. Soc. 1970. V. 25. № 4. P. 811-815.

[7] Берман С. Д. К теории групповых кодов // Кибернетика. 1967. №1. С. 31-39.

Московский государственный университет им. М.В. Ломоносова

Поступило 04.06.2001 